\title{
Fusion of intra-oral scans in cone-beam computed tomography scans
}

\author{
F. Baan ${ }^{1,2}$ (D) $\cdot$ R. Bruggink ${ }^{1,2}$ - J. Nijsink ${ }^{1}$ - T. J. J. Maal ${ }^{1,3} \cdot$ E. M. Ongkosuwito ${ }^{2,4}$
}

Received: 26 March 2020 / Accepted: 8 May 2020 / Published online: 3 June 2020

(C) The Author(s) 2020

\begin{abstract}
Purpose The purpose of this study was to evaluate the clinical accuracy of the fusion of intra-oral scans in cone-beam computed tomography $(\mathrm{CBCT})$ scans using two commercially available software packages.

Materials and methods Ten dry human skulls were subjected to structured light scanning, CBCT scanning, and intra-oral scanning. Two commercially available software packages were used to perform fusion of the intra-oral scans in the CBCT scan to create an accurate virtual head model: IPS CaseDesigner ${ }^{\circledR}$ and OrthoAnalyzer ${ }^{\mathrm{TM}}$. The structured light scanner was used as a gold standard and was superimposed on the virtual head models, created by IPS CaseDesigner® and OrthoAnalyzerTM, using an Iterative Closest Point algorithm. Differences between the positions of the intra-oral scans obtained with the software packages were recorded and expressed in six degrees of freedom as well as the inter- and intra-observer intra-class correlation coefficient. Results The tested software packages, IPS CaseDesigner® and OrthoAnalyzer ${ }^{\mathrm{TM}}$, showed a high level of accuracy compared to the gold standard. The accuracy was calculated for all six degrees of freedom. It was noticeable that the accuracy in the cranial/ caudal direction was the lowest for IPS CaseDesigner ${ }^{\circledR}$ and OrthoAnalyzer ${ }^{\mathrm{TM}}$ in both the maxilla and mandible. The inter- and intra-observer intra-class correlation coefficient showed a high level of agreement between the observers.

Clinical relevance IPS CaseDesigner ${ }^{\circledR}$ and OrthoAnalyzer ${ }^{\mathrm{TM}}$ are reliable software packages providing an accurate fusion of the intra-oral scan in the CBCT. Both software packages can be used as an accurate fusion tool of the intra-oral scan in the CBCT which provides an accurate basis for $3 \mathrm{D}$ virtual planning.
\end{abstract}

Keywords Orthognathic surgery $\cdot$ Orthodontic(s) $\cdot$ Oral implants/implantology $\cdot$ CAD $\cdot$ Treatment planning $\cdot$ Digital imaging/ radiology

\section{Introduction}

Three-dimensional (3D) virtual treatment planning is becoming an increasingly important tool within the fields of oral and

F. Baan

frank.baan@ radboudumc.nl

1 Radboudumc 3DLab The Netherlands, Radboud university medical center, Radboud Institute for Health Sciences, Geert Grooteplein Zuid 10, 6525, GA Nijmegen, The Netherlands

2 Department of Dentistry, section of Orthodontics and Craniofacial Biology, Radboud university medical center, Philips van Leydenlaan 25, 6525, EX Nijmegen, The Netherlands

3 Department of Oral and Maxillofacial Surgery, Radboud university medical center, Geert Grooteplein Zuid 10, 6525, GA Nijmegen, The Netherlands

4 Amalia Cleft and Craniofacial Centre, Radboud university medical centre, Geert Grooteplein Zuid 10, 6525, GA

Nijmegen, The Netherlands maxillofacial surgery, orthodontics, and implantology [1-3]. Complex anatomical structures and the relations between these structures can be visualized using cone-beam computed tomography $(\mathrm{CBCT})$. More specifically, bony structures and soft tissues can be captured using CBCT, which can be used to create a virtual head model $[4,5]$. In addition, 3D stereophotogrammetry can be used to add texture and detail to the virtual head model [6]. To acquire accurate information about the dentition, additional imaging of the occlusal surfaces, e.g., intra-oral scans or plaster casts, is needed because of scattering present in a CBCT due to the high density of enamel, dental restorations, implants, and orthodontic appliances [7-9]. Accurately capturing the dentition is of major importance as drilling guides, saw guides, or orthognathic positioning guides are often dental occlusal-surfacesupported [10-12]. Apart from accuracy, the dentition should also be positioned at the correct anatomical position in the mandible and maxilla. Therefore, accurate matching of the dentition in the CBCT is required for the use in clinical practice. 
Several methods are described in literature to solve the issue of a distorted occlusal area within the CBCT scan. Swennen et al. [13] proposed a triple CBCT scan method using voxel-based registration to capture accurate occlusal surfaces. However, this requires the patient to be scanned twice, increasing the radiation exposure. Other methods have investigated the image-fusion of digital dental models with the CBCT using fiducial superimposition [14-17] or surfacebased fusion $[18,19]$. The study performed by Swennen et al. [13] is, to the best knowledge of the authors, the only study that utilized commercially available software.

The use of different imaging modalities (CBCT and plaster cast/intra-oral scans) allows surgeons and technicians to virtually plan and practice several treatments before the actual treatment takes place. Three-dimensional virtual orthognathic surgical planning is a widely used tool to plan and simulate different treatment options [3]. Additionally, in dental implantology, digital implant planning is also widely used to preoperatively assess the bone quality and digitally plan the optimal implant position. Three-dimensional printed surgical drilling guides can be used to transfer the digital plan towards the operating theater [20]. Within the orthodontic work field, 3D techniques are used for several different applications. Orthodontic virtual setups can be created and used to assess the accuracy of the treatment. [21] Digitally created indirect bonding trays can be used for optimal bracket placement and an enhancement of the workflow [22].

In the current workflow, taking physical impressions, and pouring them into plaster casts, is the most used technique to capture the maxillary and mandibular occlusal surfaces precisely [23-28]. With the introduction and technical evolution of intra-oral scanners, it became easier and quicker to obtain a detailed model of the dentition of the patient without the need for physical impressions. Earlier studies proved that an intraoral scanner is a valid method for accurately visualizing the dentition. Furthermore, patients generally experience an intraoral scan as a more comfortable way of getting an accurate $3 \mathrm{D}$ dentition compared to physical impressions [28-31].

The purpose of this study is to assess the accuracy of the replacement of distorted dentition in CBCT scans with accurate digital dental models, using commercially available software.

\section{Materials and methods}

Ten dry human cadaveric skulls were obtained from the historical archive of the Radboud Anatomical Museum. For this study, no approval was required from an ethical committee. The selected cadaveric skulls had intact bony structures, $\geq 24$ teeth, and $\leq 10$ dental restorations. Dental status was recorded and summarized in Table 1.

\section{Data acquisition}

\section{Study data}

The ten dry skulls were scanned with a KaVo 3D Exam CBCT scanner (KaVo Germany) with an extended height protocol (FOV $23 \times 17 \mathrm{~cm}$ at $120 \mathrm{kV}$ and $0.4 \mathrm{~mm}$ isotropic voxel size). The skulls were scanned with a wax bite in place to ensure a centric relation. After CBCT scanning, the dentition was scanned using an intra-oral scanner (TRIOS3, 3shape A/S, Copenhagen, Denmark). The maxillary and mandibular dentition were scanned separately and exported in the Standard Tessellation Language (STL) format.

\section{Gold standard}

All ten skulls were also digitized using a structured light scanner (Scan in a box-FX, Open Technologies Srl, Italy) with an accuracy of $100 \mu \mathrm{m}$ as specified by the manufacturer. The 3D surface models were exported in the STL format. The 3D surface models contain detailed information of the bony parts of the skull as well as detailed information of the dentition and were therefore used as the gold standard in this study.

\section{Fusion}

The fusion of the dentition from the intra-oral scans in the CBCT scans was studied for two different commercial software packages. The first software package tested in this study was IPS CaseDesigner® (KLS Martin, Tuttlingen, Germany). To ensure proper fusion, the STL models of the dental arches created with the intra-oral scanner were rotated $90^{\circ}$ around the $x$-axis to align them with the coordinate system of IPS CaseDesigner®. Rotating the STL files was needed because the coordinate system used by the intra-oral scanner was not the same as the coordinate system of IPS CaseDesigner ${ }^{\circledR}$. The DICOM files of the extended height CBCT scan were imported to create a 3D virtual head model. The rotated STL models of the dental arches created were also imported. By indicating the right and left condyle, mesial cusp of the first upper right and left molar and the middle of the two upper incisors, IPS CaseDesigner ${ }^{\circledR}$ automatically aligns the STL models of the dentition with the 3D virtual head based on contrast information at the tooth crown margin (Fig. 1). After alignment, no manual adjustment was performed and the virtual head model with the incorporated intra-oral scans were exported in their correct position.

The second software package tested was the OrthoAnalyzerTM software (3shape A/S, v2019, Copenhagen, Denmark). The DICOM files of the extended height CBCT scan were imported together with the STL models of the dentition. The STL models of the dental arches were not rotated as the coordinate system between the intra-oral scanner and the 
Table 1 Dental status of the included cadaveric skulls. FDI annotation is used for tooth numbering

\begin{tabular}{|c|c|c|c|c|c|c|}
\hline Skull no. & Dental range & Missing tooth & Filled tooth & Dental range & Missing tooth & Filled tooth \\
\hline & Maxilla & & & Mandible & & \\
\hline 1 & $17-27$ & - & $16,17,26$ & $37-47$ & $36,32,46$ & 34,47 \\
\hline 2 & $17-28$ & 13,25 & - & $38-48$ & - & - \\
\hline 3 & $17-27$ & - & - & $37-47$ & - & - \\
\hline 4 & $18-28$ & $14,17,25$ & - & $38-48$ & - & - \\
\hline 5 & $18-28$ & - & 15,27 & $38-48$ & - & 37,46 \\
\hline 6 & $18-28$ & - & 16 & $38-48$ & - & 36,46 \\
\hline 7 & $17-27$ & - & 24,26 & $37-47$ & - & 33,45 \\
\hline 8 & $17-27$ & 16,23 & 15,17 & $36-47$ & 46 & 35 \\
\hline 9 & $18-28$ & - & - & $38-48$ & - & - \\
\hline 10 & $18-28$ & - & - & $38-48$ & - & - \\
\hline
\end{tabular}

OrthoAnalyzer ${ }^{\mathrm{TM}}$ software was the same. Two sets of corresponding dental landmarks (frontal incisor, left and right molar) were selected on both the 3D CBCT head model and intra-oral scans (Fig. 2). First, OrthoAnalyzer ${ }^{\mathrm{TM}}$ roughly aligned the skull model and the intra-oral scans based on the two sets of corresponding landmarks. Second and last, the shape and outline of the dentition was used for automatic alignment. No manual adjustment was performed after aligning the intra-oral scans with the CBCT model and the virtual head model intraoral scans were exported in their new position.
Fig. 1 Overview of the fusion process in the IPS

CaseDesigner®. a Three-

dimensional CBCT skull model in the IPS CaseDesigner® software with the left and the right condyle indicated as well as the occlusion plane. b Intra-oral scans of maxilla and mandible. c Crosssectional view of the 3D CBCT skull model and fused intra-oral scans. d Frontal view of the result of the fusion of the intra-oral scans in the $\mathrm{CBCT}$

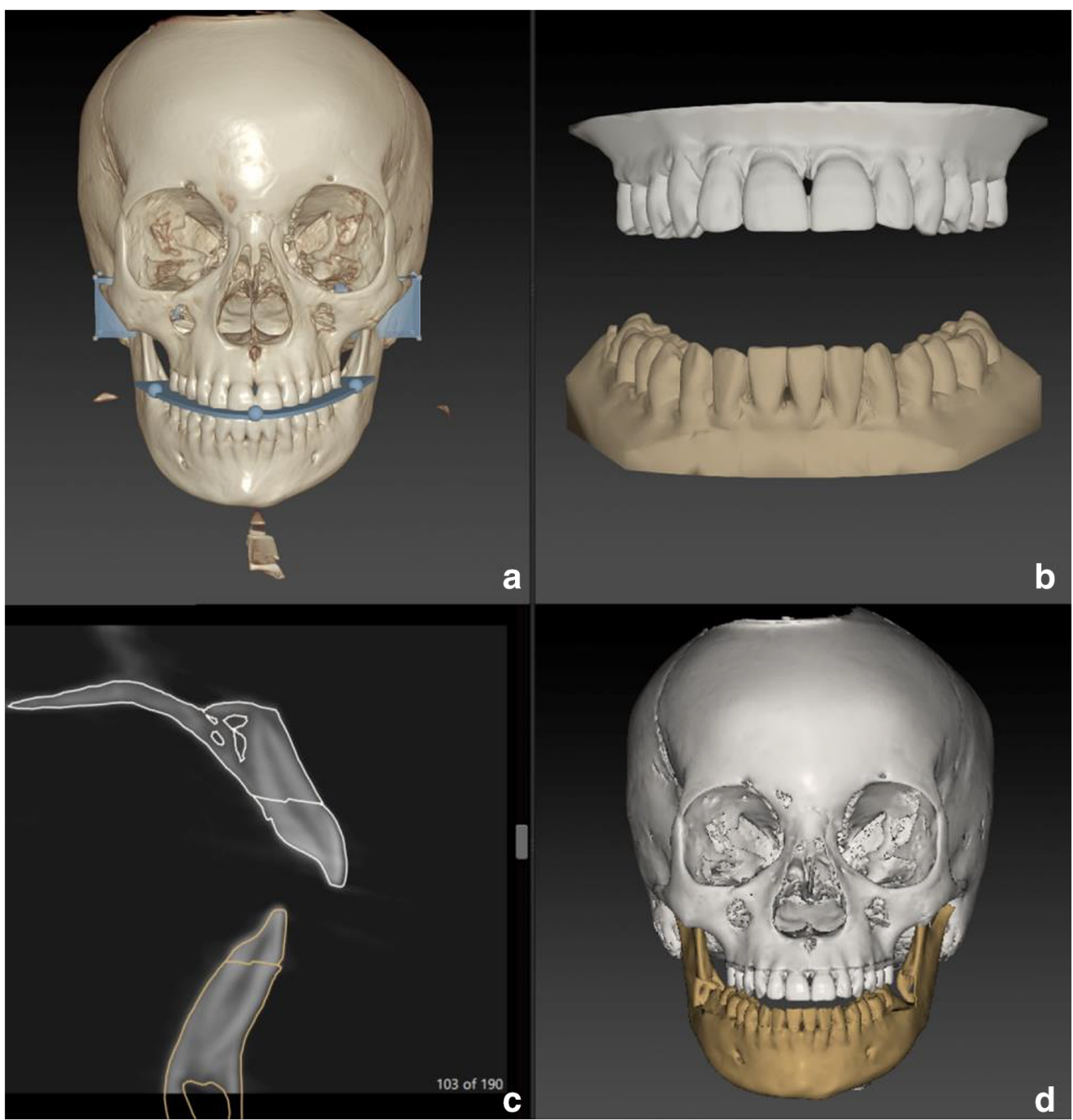


Fig. 2 Overview of the fusion process of the intra-oral scans in the CBCT in OrthoAnalyzer ${ }^{\mathrm{TM}}$. a Three-dimensional CBCT skull model in OrthoAnalyzer ${ }^{\mathrm{TM}}$ with three points on both the maxillary and mandibular teeth. b Intra-oral scans of maxilla and mandible with three points on both the maxillary and mandibular teeth, corresponding with the points shown in 3A. c Cross-sectional view of the 3D CBCT skull model and fused intra-oral scans (shown in green). d Frontal view of the result of the fusion of the intraoral scans in the $\mathrm{CBCT}$

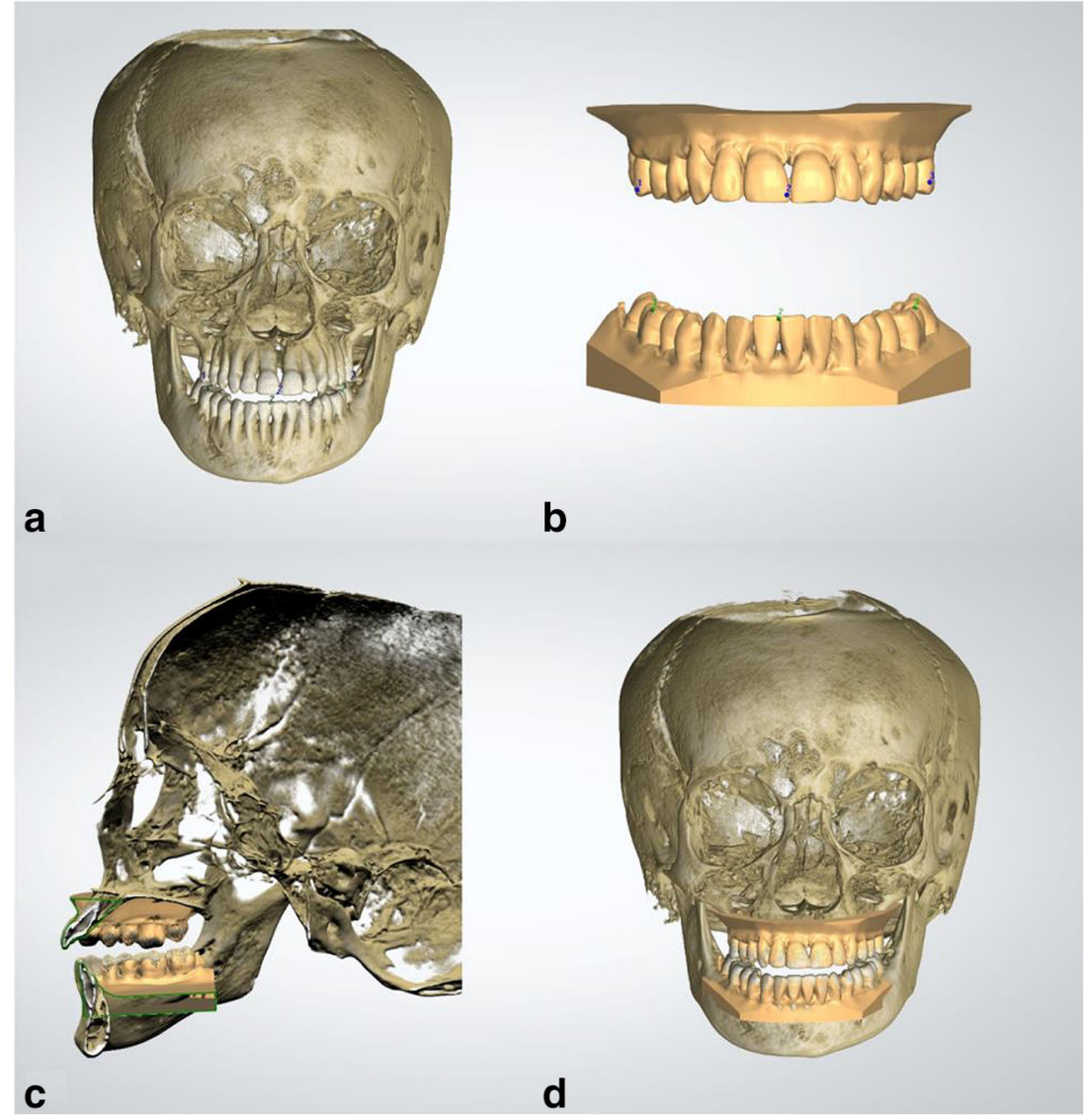

\section{Accuracy study}

To investigate the accuracy of the fusion of the intra-oral scan with the extended height CBCT scan, the first step was to align the structured light scan of the skull with the virtual head model created with IPS CaseDesigner ${ }^{\circledR}$ (Fig. 3a) and OrthoAnalyzer ${ }^{\mathrm{TM}}$ (Fig. 3c). The virtual head models created with IPS CaseDesigner® and OrthoAnalyzer ${ }^{\mathrm{TM}}$ were imported into 3DMedX (v1.2.0.4, 3D Lab Radboudumc, Nijmegen) as well as the structured light scan (Fig. 3c). An iterative closest point (ICP) algorithm [32] was used to align the different head models from the different software packages. ICP registration is an accurate and reliable method for registration of similar surfaces [32]. The algorithm enables the alignment of different 3D surfaces in such a way that the difference between the two surfaces is minimized. The intra-oral scans were incorporated in the virtual head models. The ICP algorithm was used to align the structured light scan towards the virtual head models created with IPS CaseDesigner ${ }^{\circledR}$ and OrthoAnalyzerTM using the forehead, orbital rims and zygomatic area as reference (Fig. 3c, d). Distance maps were calculated to assess the accuracy of the alignment of the virtual head models with the structured light scan (gold standard).
Finally, the intra-oral scans from the OrthoAnalyzer ${ }^{\mathrm{TM}}$ and IPS CaseDesigner ${ }^{\circledR}$ were displaced towards the structured light scanned dentition using the ICP algorithm (Fig. 3b). The ICP algorithm aligns the different surfaces and also provides a transformation matrix of the exact displacement needed to perfectly align the intra-oral scans from the OrthoAnalyzer ${ }^{\mathrm{TM}}$ and IPS CaseDesigner ${ }^{\circledR}$ towards the structured light scanned dentition. The transformation matrix contains the translational and rotational directions. The transformation matrix was converted to clinically relevant information using the same method as described by LaValle [33]. The translations and rotations were recorded and converted to the six degrees of freedom (DOF) for the dentition of the maxilla and the dentition of the mandible (Fig. 3h, i).

\section{Statistics}

Data was tested for normality using the Shapiro-Wilk test for small sample size. A paired sample $t$ test was used to evaluate the accuracy of the placement of the intra-oral scan in the CBCT for both IPS CaseDesigner ${ }^{\circledR}$ and OrthoAnalyzerTM To assess the reproducibility of the fusion, two experienced observers repeated the fusion in both the IPS CaseDesigner® and the OrthoAnalyzer ${ }^{\mathrm{TM}}$ software for all ten dry skulls. The intra- and inter-observer reproducibility of the fusion for 


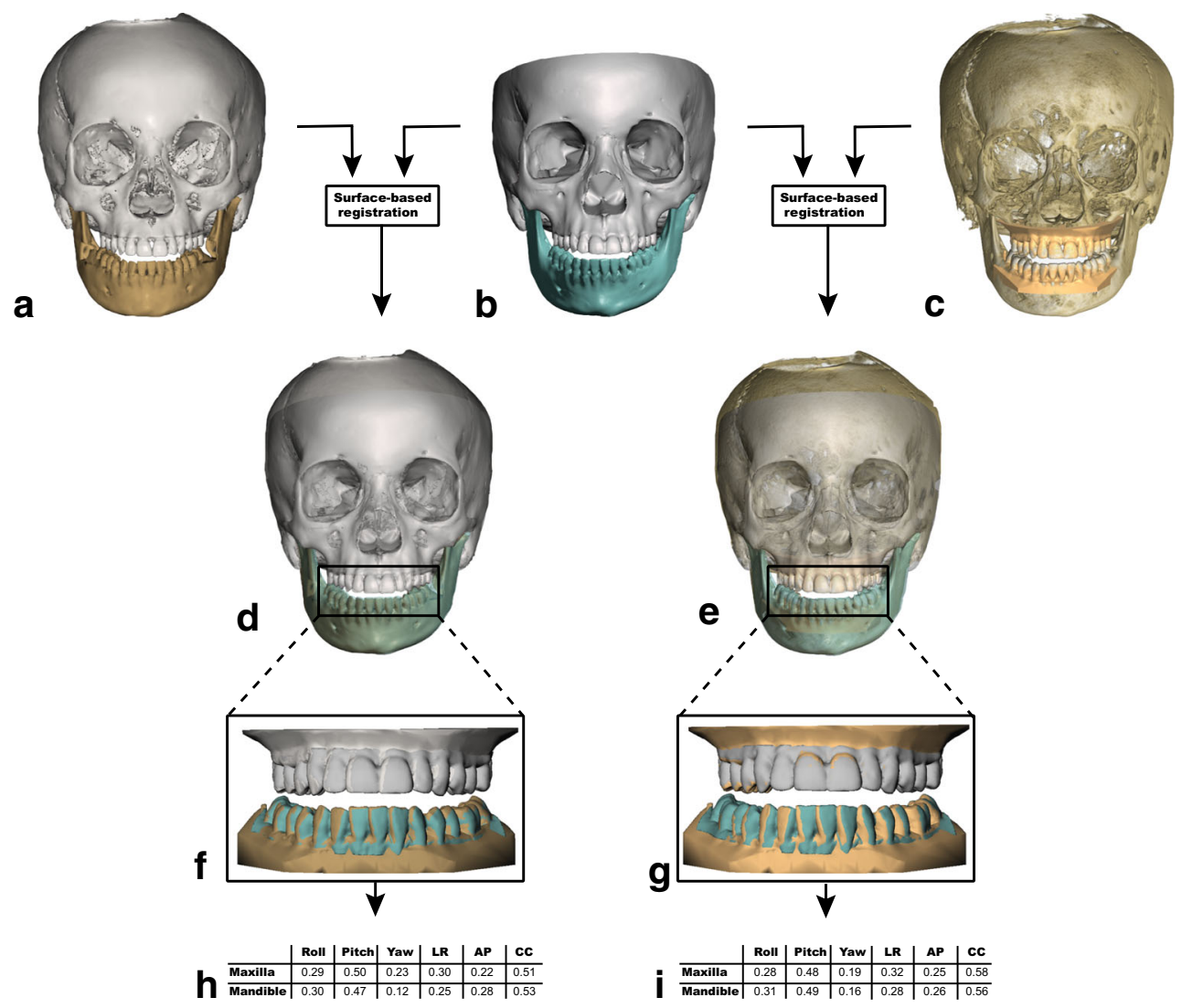

Fig. 3 Overview of the fusion process. (A) Virtual head model made with IPS CaseDesigner®. (B) Structured light scan (gold standard) of a dry humal skull with high detail of the bony structures as well as the teeth. (C) Virtual head model made with OrthoAnalyzer ${ }^{\mathrm{TM}}$. (D) Superimposition of the gold standard with the virtual head model created in IPS CaseDesigner ${ }^{\circledR}$ using the forehead, orbital rims, and zygomatic area as reference. (E) Superimposition of the gold standard with the virtual head model created in OrthoAnalyzer ${ }^{\mathrm{TM}}$ using the forehead, orbital rims, and zygomatic area as reference. (F) The different positions of the dental arches of IPS CaseDesigner® and the gold standard. Blue and gray:

dental arch position of the gold standard, brown and white: position of the dental arches fused using IPS CaseDesigner®. (G) The different positions of the dental arches of OrthoAnalyzer ${ }^{\mathrm{TM}}$ and the gold standard. Blue and gray: dental arch position of the gold standard, brown: position of the dental arches fused using IPS CaseDesigner®. (H) Differences of the dental arches expressed in six DOF for IPS CaseDesigner® and the gold standard for both the maxilla and mandible. (I) Differences of the dental arches expressed in six DOF for OrthoAnalyzer ${ }^{\mathrm{TM}}$ and the gold standard for both the maxilla and mandible. LR left/right, AP anterior/ posterior, CC cranial/caudal

OrthoAnalyzer ${ }^{\mathrm{TM}}$ and IPS CaseDesigner ${ }^{\circledR}$ were assessed using the intraclass correlation coefficient. For all tests, the significance level was set at $p<0.05$.

\section{Results}

A total of ten dry skulls were used in the study. All skulls had $\geq 24$ teeth in order to achieve a proper fusion of the intra-oral scan in the CBCT. Data distribution was tested for normality using the Shapiro-Wilk test and showed a normal distribution $(p>0.05)$.

\section{Gold standard}

Aligning the structured light scan (gold standard) towards the CBCT using the ICP algorithm in the 3DMedX software, using the forehead, orbital rims and zygomatic area as

reference, resulted in an average matching accuracy of 0.20 $\pm 0.16 \mathrm{~mm}$ for the skull/maxilla and $0.11 \pm 0.12 \mathrm{~mm}$ for the mandible.

\section{Accuracy}

The translational directions investigated were left/right, anterior/posterior, and cranial/caudal. For the rotational directions, the Pitch (rotation around the $x$-axis), Roll (rotation around $y$ axis), and Yaw (rotation around $z$-axis) rotations were used. Translational and rotational directions are displayed in Table 2 for both IPS CaseDesigner ${ }^{\circledR}$ and OrthoAnalyzerTM Corresponding $p$ values are also given in Table 1 as well as the $p$ values for the statistical differences between IPS CaseDesigner ${ }^{\circledR}$ and OrthoAnalyzer ${ }^{\mathrm{TM}}$.

For IPS CaseDesigner®, translational differences compared to the gold standard were all smaller than the voxel size of the CBCT $(0.40 \mathrm{~mm})$ except for the cranial/caudal direction 
which was the largest in the mandible $(0.45 \pm 0.46 \mathrm{~mm})$. Rotational differences compared to the gold standard did not exceed $0.32^{\circ}$ with the yaw of mandible showing the smallest difference $\left(0.16 \pm 0.12^{\circ}\right)$ and the roll of the maxilla the largest $\left(0.29 \pm 0.28^{\circ}\right)$. The paired $t$ test showed a statistically significant difference between the gold standard and IPS CaseDesigner ${ }^{\circledR}$ for all translations and rotations. However, the differences were $\leq 0.40 \mathrm{~mm}$ except for the cranial-caudal translation.

For the OrthoAnalyzer ${ }^{\mathrm{TM}}$ software, the translational differences were the largest for cranial/caudal direction of the maxilla $(0.83 \pm 0.54 \mathrm{~mm})$ whereas the left/right direction of the mandible showed the smallest inaccuracy $(0.41 \pm 0.44 \mathrm{~mm})$. Rotational differences did not exceed $0.45^{\circ}$, with the roll of the mandible showing the smallest difference $\left(0.21 \pm 0.21^{\circ}\right)$ and the roll of the maxilla the largest $\left(0.45 \pm 0.35^{\circ}\right)$. Translational and rotational differences all statistically differ from the gold standard for the OrthoAnalyzer ${ }^{\mathrm{TM}}$.

IPS CaseDesigner® showed the smallest overall difference for the maxilla $(0.60 \mathrm{~mm})$ whereas the maxilla of the OrthoAnalyzer ${ }^{\mathrm{TM}}$ showed the largest difference $(1.18 \mathrm{~mm})$.

Comparing both software packages, OrthoAnalyzer ${ }^{\mathrm{TM}}$ showed bigger translational discrepancies compared to IPS CaseDesigner®. Only the left/right direction of the mandible $(p=0.01)$ showed a statistically significant difference. Rotational differences were all smaller than $0.50^{\circ}$ for all ten skulls, and only the Roll of the maxilla showed a statistically significant difference $(p=0.04)$.

\section{Reproducibility}

The intra-observer overall mean difference was $0.08 \pm$ $0.10 \mathrm{~mm}$ for IPS CaseDesigner ${ }^{\circledR}$ and $0.18 \pm 0.15 \mathrm{~mm}$ for OrthoAnalyzer ${ }^{\mathrm{TM}}$. The inter-observer overall mean difference was $0.11 \pm 0.12 \mathrm{~mm}$ for IPS CaseDesigner® and $0.21 \pm$ $0.19 \mathrm{~mm}$ for OrthoAnalyzer ${ }^{\mathrm{TM}}$. The intra- and inter-observer reliability analysis showed good correlation and intraclass coefficients for OrthoAnalyzer ${ }^{\mathrm{TM}}$. High correlation and intraclass coefficients were found for IPS CaseDesigner®. In comparison with IPS CaseDesigner ${ }^{\circledR}$, OrthoAnalyzer ${ }^{\mathrm{TM}}$ showed lower scores. The results of the analysis are shown in Table 3.

\section{Discussion}

CBCT imaging is a widely used tool for capturing the human skull. However, CBCT has the drawback that it is prone to distortions around the dentition. Metallic restorations, orthodontic appliances, and the high density of enamel cause distortion of the dentition in the CBCT model [7-9]. In order to utilize $\mathrm{CBCT}$ imaging for $\mathrm{CAD} / \mathrm{CAM}$ processes, additional imaging is needed as well as a proper fusion between the 
Table 3 The intraclass correlation coefficient displayed for all translations and rotations for both the maxilla and mandible

\begin{tabular}{lllllllll}
\hline & & LR & AP & CC & Roll & Pitch & Yaw \\
\hline \multirow{2}{*}{ IPS Case Designer® } & \multirow{2}{*}{ Maxilla } & Intra-observer ICC & 0.97 & 0.98 & 0.97 & 0.93 & 0.95 & 0.97 \\
& \multirow{4}{*}{ Mandible } & Inter-observer ICC & 0.94 & 0.98 & 0.92 & 0.92 & 0.97 & 0.90 \\
& & Intra-observer ICC & 0.99 & 0.99 & 0.98 & 0.99 & 0.98 & 0.97 \\
& Inter-observer ICC & 0.97 & 0.97 & 0.93 & 0.99 & 0.99 & 0.96 \\
Ortho AnalyzerTM & \multirow{2}{*}{ Maxilla } & Intra-observer ICC & 0.90 & 0.84 & 0.89 & 0.89 & 0.83 & 0.92 \\
& \multirow{3}{*}{ Mandible } & Inter-observer ICC & 0.84 & 0.85 & 0.82 & 0.88 & 0.88 & 0.90 \\
& & Intra-observer ICC & 0.89 & 089 & 0.92 & 0.90 & 0.92 & 0.93 \\
& & Inter-observer ICC & 0.88 & 0.84 & 0.89 & 0.90 & 0.90 & 0.93 \\
\hline
\end{tabular}

ICC intraclass correlation coefficient different imaging modalities. In a recent review of Mangano et al. [34], it was concluded that there was still no easy way to fuse scans from different image modalities.

In this study, the accuracy of the fusion of intra-oral scans into CBCT models was assessed using commercially available software. The tested software packages, IPS CaseDesigner® and OrthoAnalyzer ${ }^{\mathrm{TM}}$, showed a high level of accuracy compared to the gold standard. The accuracy was calculated for all six degrees of freedom. It was noticeable that the accuracy in the cranial/caudal direction was the lowest for IPS CaseDesigner $®$ and OrthoAnalyzer ${ }^{\mathrm{TM}}$ in both the maxilla and mandible. A logical reason for this lower accuracy could not be found, but it is worth to note this difference. The user of these software packages should take this larger inaccuracy in the cranial/caudal direction into account when performing a surgical planning. A visual check whether the software performed an accurate fusion is strongly advisable.

An important step in assessing the fusion accuracy is the alignment of the structured light scan of the skull (gold standard) with the CBCT 3D model. This was performed utilizing a validated ICP algorithm [32]. The accuracy of the alignment of the gold standard with the CBCT 3D model was $0.20 \mathrm{~mm}$. This is a clinically acceptable result as the resolution of the CBCT scans was $0.40 \mathrm{~mm}$.

Overall mean intra- and inter-observer differences were low $(\leq 0.21 \mathrm{~mm})$ which was reflected in the ICC values found. In the OrthoAnalyzer ${ }^{\mathrm{TM}}$, the user needs to provide three corresponding points on both the CBCT 3D model and the intra-oral scan. As the results show, this increases the intra- or inter-observer variability in OrthoAnalyzer ${ }^{\mathrm{TM}}$ compared to the workflow in IPS CaseDesigner which did not use additional manual input. However, all ICC values were $>0.82$ showing good agreement for OrthoAnalyzer ${ }^{\mathrm{TM}}$ and $>0.92$ for IPS CaseDesigner® showing excellent intraand inter-observer agreement.

When comparing the fusion techniques of this current study to earlier studies, it is noteworthy that all earlier studies either utilize intra-oral markers [17, 35, 36], extra-oral markers [15, 37], or utilized a double/triple scanning procedure [13]. The fusion technique utilized by IPS CaseDesigner ${ }^{\circledR}$ and OrthoAnalyzer ${ }^{\mathrm{TM}}$ does not require markers or an additional CBCT scan. This makes it a convenient method for use in the clinical practice. Moreover, the accuracy found for IPS CaseDesigner® is in line with other studies. A splint with ceramic balls was used by Uechi et al. [15], and a root-mean-square error of $0.4 \mathrm{~mm}$ was found. Another study found an accuracy ranging from 0.10 to $0.50 \mathrm{~mm}$ by using titanium markers [37]. De Waard et al. found errors ranging from 0.12 to $0.45 \mathrm{~mm}$. Another study performed by Lin et al. using surface-based matching found errors ranging from 0.11 to $0.53 \mathrm{~mm}$. However, most of these studies assessed the accuracy of the fusion (e.g., how do the markers overlap) instead of assessing the differences using a true gold standard. The accuracies found for IPS CaseDesigner are in the range of these studies as the overall accuracy is $\leq 0.66 \mathrm{~mm}$. OrthoAnalyzer shows bigger discrepancies $(\leq 1.18 \mathrm{~mm})$ and is therefore not in line with earlier studies.

A limitation of the current study is the use of dry human skulls which could influence the result of the study. For example, accuracy of intra-oral scanning can be lower in actual patients as patient movement, anatomical restrictions, and excessive saliva can hamper proper imaging of the dentition [28]. Another limitation of the current study was the absence of orthodontic appliances. Most orthognathic patients have orthodontic brackets which can influence the accuracy of the fusion as might cause distortion of the CBCT scan. Therefore, a future study to investigate the effects of orthodontic appliances is necessary. Furthermore, to study the use of the fusion of intra-oral scans in CBCT in implantology patients closer, a study should be designed in which partial dental arches are used to see whether the fusion is still accurate if more teeth are missing.

Recent developments in artificial intelligence (AI) are promising. Earlier studies showed that using AI, it is possible to segment third molars from an orthopantomogram [38]. With future developments, AI might be a promising technique to automatically "recognize" dentition in a CBCT image. 
Recognizing the dentition might make it easier to replace it and therefore enhancing the accuracy of the fusion between intra-oral scans and CBCT. As AI (e.g., convolutional neural networks) is an upcoming and promising technique in image fusion [39], development of AI-driven algorithms to fuse dental information with CBCT data might result in a more accurate and automated solution.

\section{Conclusion}

IPS CaseDesigner ${ }^{\circledR}$ is a reliable software packages within the scope of this study. It provides accurate fusion of the intra-oral scan in the CBCT when a complete dental arch is used with little to none dental fillings. OrthoAnalyzer ${ }^{\mathrm{TM}}$ showed bigger discrepancies, and therefore, it is recommended to perform proper visual inspection before using the fusion. Inaccuracies were found in both packages. However, for IPS CaseDesigner, these are in line with the findings of similar studies. OrthoAnalyzer shows bigger discrepancies. Future research towards the effect of scattering caused by fillings and orthodontic appliances is recommended as well as the influence of missing teeth.

Author contributions F. Baan contributed to the conception, design, data digitalization, data analysis, interpretation, and drafted and critically revised the manuscript. R. Bruggink contributed to the design, data analysis, interpretation, and drafted and critically revised the manuscript. J. Nijsink contributed to the design, data digitalization, data analysis, and drafted and critically revised the manuscript. E.M. Ongkosuwito and T.J.J. Maal contributed to the conception, design, data interpretation, and critically revised the manuscript. All authors gave final approval and agree to be accountable for all aspects of the work.

\section{Compliance with ethical standards}

Conflict of interest The authors declare that they have no conflict of interest.

Ethical approval For this study, no approval was required from an ethical committee.

Informed Consent For this type of study, formal consent is not required.

Open Access This article is licensed under a Creative Commons Attribution 4.0 International License, which permits use, sharing, adaptation, distribution and reproduction in any medium or format, as long as you give appropriate credit to the original author(s) and the source, provide a link to the Creative Commons licence, and indicate if changes were made. The images or other third party material in this article are included in the article's Creative Commons licence, unless indicated otherwise in a credit line to the material. If material is not included in the article's Creative Commons licence and your intended use is not permitted by statutory regulation or exceeds the permitted use, you will need to obtain permission directly from the copyright holder. To view a copy of this licence, visit http://creativecommons.org/licenses/by/4.0/.

\section{References}

1. Jheon AH, Oberoi S, Solem RC, Kapila S (2017) Moving towards precision orthodontics: An evolving paradigm shift in the planning and delivery of customized orthodontic therapy. Orthod. Craniofacial Res. 20(March):106-113

2. Verhamme LM, Meijer GJ, Bergé SJ, Soehardi RA, Xi T, de Haan AFJ, Schutyser F, Maal TJJ (2015) An accuracy study of computerplanned implant placement in the augmented maxilla using mucosa-supported surgical templates. Clin Implant Dent Relat Res 17(6):1154-1163

3. Stokbro K, Aagaard E, Torkov P, Bell RB, Thygesen T (2014) Virtual planning in orthognathic surgery. Int J Oral Maxillofac Surg 43(8):957-965

4. Weiss R, Read-Fuller A (2019) Cone beam computed tomography in oral and maxillofacial surgery: an evidence-based review. Dent $\mathrm{J}$ $7(2): 1-23$

5. Liebregts J, Xi T, Timmermans M, de Koning M, Bergé S, Hoppenreijs T, Maal T (2015) Accuracy of three-dimensional soft tissue simulation in bimaxillary osteotomies. J Craniomaxillofac Surg 43(3):329-335

6. Verhulst A, Hol M, Vreeken R, Becking A, Ulrich D, Maal T (2018) Three-dimensional imaging of the face: a comparison between three different imaging modalities. Aesthetic Surg J 38(6): $579-585$

7. Hirschinger V, Hanke S, Hirschfelder U, Hofmann E (2015) Artifacts in orthodontic bracket systems in cone-beam computed tomography and multislice computed tomography. J Orofac Orthop 76(2):152-163

8. Schulze RKW, Berndt D, d'Hoedt B (2010) On cone-beam computed tomography artifacts induced by titanium implants. Clin Oral Implants Res 21(1):100-107

9. Schulze R, Heil U, Gro $\beta$ D, Bruellmann DD, Dranischnikow E, Schwanecke U, Schoemer E (2011) Artefacts in CBCT: a review. Dentomaxillofacial Radiol. 40(5):265-273

10. Savoldelli C, Vandersteen C, Dassonville O, Santini J (2018) Dental occlusal-surface-supported titanium guide to assist cutting and drilling in mandibular bilateral sagittal split osteotomy. J Stomatol Oral Maxillofac Surg 119(1):75-78

11. Polley JW, Figueroa AA (2013) Orthognathic positioning system: intraoperative system to transfer virtual surgical plan to operating field during orthognathic surgery. J Oral Maxillofac Surg 71(5): 911-920

12. Flügge TV, Nelson K, Schmelzeisen R, Metzger MC (2013) Threedimensional plotting and printing of an implant drilling guide: simplifying guided implant surgery. J Oral Maxillofac Surg 71(8): 1340-1346

13. Swennen GRJ, Mollemans W, de Clercq C, Abeloos J, Lamoral P, Lippens F, Neyt N, Casselman J, Schutyser F (2009) A cone-beam computed tomography triple scan procedure to obtain a threedimensional augmented virtual skull model appropriate for orthognathic surgery planning. J. Craniofac. Surg. 20(2):297-307

14. Gateno J, Xia J, Teichgraeber JF, Rosen A (2003) A new technique for the creation of a computerized composite skull model. J Oral Maxillofac Surg 61(2):222-227

15. Uechi J, Okayama M, Shibata T, Muguruma T, Hayashi K, Endo K, Mizoguchi I (2006) A novel method for the 3-dimensional simulation of orthognathic surgery by using a multimodal image-fusion technique. Am J Orthod Dentofac Orthop 130(6):786-798

16. Sun Y, Luebbers HT, Agbaje JO, Schepers S, Vrielinck L, Lambrichts I, Politis C (2013) Accuracy of upper jaw positioning with intermediate splint fabrication after virtual planning in bimaxillary orthognathic surgery. J Craniofac Surg 24(6):18711876 
17. Almutairi T, Naudi K, Nairn N, Ju X, Whitters J, Ayoub A (2018) Replacement of the distorted dentition of the cone-beam computed tomography scans for orthognathic surgery planning. J. Oral Maxillofac. Surg. 76(7):1561.e1-1561.e8

18. Nkenke E, Zachow S, Benz M, Maier T, Veit K, Kramer M, Benz S, Häusler G, Neukam FW, Lell M (2004) Fusion of computed tomography data and optical 3D images of the dentition for streak artefact correction in the simulation of orthognathic surgery. Dentomaxillofacial Radiol 33(4):226-232

19. Lin HH, Chiang WC, Lo LJ, Sheng-Pin Hsu S, Wang CH, Wan SY (Nov. 2013) Artifact-resistant superimposition of digital dental models and cone-beam computed tomography images. J Oral Maxillofac Surg 71(11):1933-1947

20. Chiarelli T, Franchini F, Lamma A, Lamma E, Sansoni T (2011) From implant planning to surgical execution: an integrated approach for surgery in oral implantology. Int. J. Med. Robot. Comput. Assist. Surg. 8(October):57-66

21. F. Baan, O. de Waard, R. Bruggink, T. Xi, E. M. Ongkosuwito, and T. J. J. Maal, "Virtual setup in orthodontics: planning and evaluation," Clin. Oral Investig., 2019

22. de Oliveira NS, Gribel BF, Neves LS, Lages EMB, Macari S, Pretti $\mathrm{H}$ (2019) Comparison of the accuracy of virtual and direct bonding of orthodontic accessories. Dental Press J Orthod 24(4):46-53

23. Fleming PS, Marinho V, Johal A (2011) Orthodontic measurements on digital study models compared with plaster models: a systematic review. Orthod Craniofacial Res 14(1):1-16

24. Nadjmi N, Mollemans W, Daelemans A, Van Hemelen G, Schutyser F, Bergé S (2010) Virtual occlusion in planning orthognathic surgical procedures. Int J Oral Maxillofac Surg 39(5):457-462

25. Wiranto MG, Engelbrecht WP, Tutein Nolthenius HE, Van Der Meer WJ, Ren Y (2013) Validity, reliability, and reproducibility of linear measurements on digital models obtained from intraoral and cone-beam computed tomography scans of alginate impressions. Am J Orthod Dentofac Orthop 143(1):140-147

26. De Luca Canto G, Pachêco-Pereira C, Lagravere MO, Flores-Mir C, Major PW (2015) Intra-arch dimensional measurement validity of laser-scanned digital dental models compared with the original plaster models: a systematic review. Orthod Craniofac Res 18(2):65-76

27. Plooij JM, Maal TJJ, Haers P, Borstlap WA, Kuijpers-Jagtman AM, Bergé SJ (2011) Digital three-dimensional image fusion processes for planning and evaluating orthodontics and orthognathic surgery. A systematic review. Int J Oral Maxillofac Surg 40(4): 341-352

28. Zhang F, Suh KJ, Lee KM (2016) Validity of intraoral scans compared with plaster models: An in-vivo comparison of dental measurements and 3D surface analysis. PLoS One 11(6):157713
29. Yuzbasioglu E, Kurt H, Turunc R, Bilir H (2014) Comparison of digital and conventional impression techniques: evaluation of patients' perception, treatment comfort, effectiveness and clinical outcomes. BMC Oral Health 14(1):10

30. Londono J, Abreu A, Baker PS, Furness AR (2015) Fabrication of a definitive obturator from a $3 \mathrm{D}$ cast with a chairside digital scanner for a patient with severe gag reflex: a clinical report. J Prosthet Dent 114(5):735-738

31. Wismeijer D, Mans R, van Genuchten M, Reijers HA (2014) Patients' preferences when comparing analogue implant impressions using a polyether impression material versus digital impressions (intraoral scan) of dental implants. Clin Oral Implants Res 25(10):1113-1118

32. Besl PJ, McKay HD (1992) A method for registration of 3-D shapes. IEEE Trans Pattern Anal Mach Intell 14(2):239-256

33. S. M. LaValle, Planning algorithms. Cambridge University Press, 2006

34. Mangano C, Luongo F, Migliario M, Mortellaro C, Mangano FG (2018) Combining intraoral scans, cone beam computed tomography and face scans: the virtual patient. J. Craniofac. Surg. 29(8): 2241-2246

35. Swennen GRJ, Mommaerts MY, Abeloos J, de Clercq C, Lamoral P, Neyt N, Casselman J, Schutyser F (Jan. 2009) A cone-beam CT based technique to augment the $3 \mathrm{D}$ virtual skull model with a detailed dental surface. Int J Oral Maxillofac Surg 38(1):48-57

36. De Waard O, Baan F, Verhamme L, Breuning H, Kuijpers-Jagtman AM, Maal T (Feb. 2016) A novel method for fusion of intra-oral scans and cone-beam computed tomography scans for orthognathic surgery planning. J Cranio-Maxillofacial Surg 44(2):160-166

37. Gateno J, Xia JJ, Teichgraeber JF, Christensen AM, Lemoine JJ, Liebschner MAK, Gliddon MJ, Briggs ME (Apr. 2007) Clinical feasibility of computer-aided surgical simulation (CASS) in the treatment of complex cranio-maxillofacial deformities. J Oral Maxillofac Surg 65(4):728-734

38. Vinayahalingam S, Xi T, Bergé S, Maal T, de Jong G (2019) Automated detection of third molars and mandibular nerve by deep learning. Sci Rep 9(1):1-7

39. Dubost F, Bruijne M, Nardin M, Dalca AV, Donahue KL, Giese AK, Etherton MR, Wu O, Groot M, Niessen W, Vernooij M, Rost NS, Schirmer MD (2020) Multi-atlas image registration of clinical data with automated quality assessment using ventricle segmentation. Med Image Anal 63:101698

Publisher's note Springer Nature remains neutral with regard to jurisdictional claims in published maps and institutional affiliations. 